\title{
Metal-polymer hybrid nanomaterials for plasmonic ultrafast hydrogen detection
}

\section{Nugroho, Ferry A.A.; Darmadi, Iwan; Cusinato, Lucy; Susarrey-Arce, Arturo; Schreuders, Herman; Bannenberg, Lars J.; Bastos da Silva Fanta, Alice; Kadkhodazadeh, Shima; Wagner, Jakob Birkedal; Antosiewicz, Tomasz J.}

Total number of authors:

14

Published in:

Nature Materials

Link to article, DOI:

10.1038/s41563-019-0325-4

Publication date:

2019

Document Version

Peer reviewed version

Link back to DTU Orbit

Citation (APA):

Nugroho, F. A. A., Darmadi, I., Cusinato, L., Susarrey-Arce, A., Schreuders, H., Bannenberg, L. J., Bastos da Silva Fanta, A., Kadkhodazadeh, S., Wagner, J. B., Antosiewicz, T. J., Hellman, A., Zhdanov, V. P., Dam, B., \& Langhammer, C. (2019). Metal-polymer hybrid nanomaterials for plasmonic ultrafast hydrogen detection. Nature Materials, 18, 489-495. https://doi.org/10.1038/s41563-019-0325-4

\section{General rights}

Copyright and moral rights for the publications made accessible in the public portal are retained by the authors and/or other copyright owners and it is a condition of accessing publications that users recognise and abide by the legal requirements associated with these rights.

- Users may download and print one copy of any publication from the public portal for the purpose of private study or research.

- You may not further distribute the material or use it for any profit-making activity or commercial gain

- You may freely distribute the URL identifying the publication in the public portal 


\section{Plasmonic Metal - Polymer Hybrid Nanomaterials for Ultrafast Hydrogen Detection}

Ferry A. A. Nugroho*, ${ }^{*}$, Iwan Darmadi ${ }^{\dagger}$, Lucy Cusinato ${ }^{\dagger}$, Arturo Susarrey-Arce ${ }^{\dagger}$, Herman Schreuders ${ }^{\sharp}$, Lars J. Bannenberg ${ }^{\ddagger}$, Alice Bastos da Silva Fanta ${ }^{\S}$, Shima Kadkhodazadeh ${ }^{\S}$, Jakob B. Wagner ${ }^{\S}$, Tomasz J. Antosiewicz ${ }^{\dagger, \perp}$, Anders Hellman ${ }^{\dagger}$, Vladimir P. Zhdanov ${ }^{\dagger, \|}$, Bernard Dam and Christoph Langhammer*,ं

${ }^{\dagger}$ Department of Physics, Chalmers University of Technology, 41296 Göteborg, Sweden.

tDepartment of Chemical Engineering, Delft University of Technology, 2629 HZ Delft, The Netherlands.

${ }^{\S}$ Center for Electron Nanoscopy, Technical University of Denmark, 2800 Kongens Lyngby, Denmark.

${ }^{\perp}$ Faculty of Physics, University of Warsaw, Pasteura 5, 02-093, Warsaw, Poland.

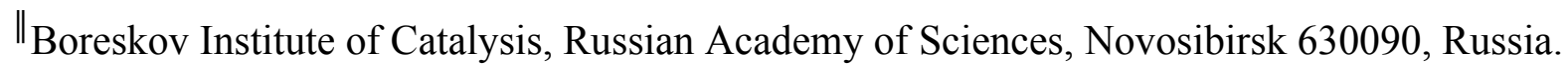

*Correspondence to: ferryn@chalmers.se, clangham@chalmers.se 


\begin{abstract}
:
Hydrogen-air mixtures are highly flammable. Therefore, hydrogen sensors are of paramount importance for timely leak detection during hydrogen handling and in a future hydrogen economy. However, none of the existing solutions meets the stringent performance targets set by stakeholders. Furthermore, deactivation due to poisoning by e.g. carbon monoxide is a widely unsolved problem. Here we present a plasmonic metal-polymer hybrid nanomaterial concept that makes it possible to reduce the apparent activation energy of hydrogen sorption in plasmonic nanoparticles by engineering the particle-polymer interface, and that provides unprecedented deactivation resistance via a tailored tandem polymer membrane. In concert with an optimized volume/surface ratio of the signal transducer uniquely offered by nanoparticles, this enables subsecond sensor response times. Simultaneously, hydrogen sorption hysteresis is suppressed, sensor limit of detection is enhanced, and sensor operation in demanding chemical environments is enabled, without signs of long-term deactivation. In a wider perspective, our work opens the door to next-generation optical gas sensors with functionalities optimized by hybrid material engineering.
\end{abstract}




\section{Main Text:}

In a hydrogen economy, ${ }^{1}$ where hydrogen gas is the clean and sustainable carrier of energy, hydrogen sensors will play a critical role due to hydrogen's wide flammability range in air. For safety reasons, any leaks in hydrogen energy storage systems, vehicles and appliances, as well as the entire hydrogen distribution infrastructure, must be detected immediately. Hence, hydrogen sensor performance targets specify, among other, a response time of $1.0 \mathrm{~s}$ at room temperature, across the concentration range from $0.1 \%$ to $10 \%{ }^{2}$ (see corresponding discussion in Supplementary Information). In the quest to meet these challenging targets, optical nanoplasmonic hydrogen sensors based on hydride-forming metal nanoparticles have been introduced. $^{3-7}$ They are attractive because the optical signals generate no sparks and stem from absorption of hydrogen species into interstitial sites of the metal host, which renders such sensors intrinsically highly hydrogen-selective. ${ }^{3}$ Furthermore, their optical fingerprint is spectrally tunable, ${ }^{4,8,9}$ and they can be miniaturized down to the single nanoparticle level. ${ }^{6,10,11}$ In this field, like in many other hydrogen sensor platforms, ${ }^{12,13} \mathrm{Pd}$ is the functional material of choice. This is due to its ability to dissociate hydrogen gas efficiently at ambient conditions, and its reversible phase transformation from metal to metal hydride at room temperature, ${ }^{3,14}$ which gives rise to a sizable optical contrast. ${ }^{15}$ However, among several other drawbacks, such as hysteretic behavior $^{16}$ and response times falling short of the target value, ${ }^{13,17}$ the problem that hydrogen dissociation on $\mathrm{Pd}$ is effectively poisoned even by trace amounts of species like $\mathrm{CO}$ and $\mathrm{NO}_{2}{ }^{17,18}$ remains widely unsolved, in particular in combination with other critical sensor metrics (Tables $\mathrm{S} 1-3)$. This is problematic since e.g. $\mathrm{CO}$ is omnipresent at ca. $0.2 \mathrm{ppm}$, and at even higher concentrations close to large point sources or in urban areas. ${ }^{19}$

The plasmonic metal-polymer optical hydrogen sensor platform presented here overcomes all these long-standing limitations by capitalizing on generic and synergistic effects that arise from combining PdAu alloy plasmonic nanoparticle signal transducers with tailored thin polymer membrane layers (Fig. 1a). As we show below, the stringent $1.0 \mathrm{~s}$ room temperature response time target can be met by tailoring the nanoparticle surface/volume ratio in concert with a reduction of the apparent activation energy by engineering the particle-polymer interface. Simultaneously, the hysteresis is suppressed, the sensor limit of detection is significantly enhanced and sensor operation in demanding chemical environments without any signs of deactivation, even after 4 months, is enabled. 


\section{Plasmonic metal-polymer optical hydrogen sensor platform}

The nanoarchitecture of our hybrid sensor consists of a nanofabricated quasi-random array of hydride-forming and plasmonically active metal nanoparticles on a glass substrate coated with a thin polymeric film (Fig. 1a-b). Specifically, we chose a $\mathrm{Pd}_{70} \mathrm{Au}_{30}$ alloy, as well as a pure $\mathrm{Pd}$ control as detector. This alloy composition is optimal because at a lower $\mathrm{Au}$ fraction sensor accuracy is compromised by hysteresis ${ }^{20,21}$ (i.e. the readout is nonspecific as it depends on the hydrogen pressure history), while at a higher $\mathrm{Au}$ fraction the sensitivity is reduced. ${ }^{4,22} \mathrm{We}$ nanofabricated the alloy and Pd nanostructures with average nanoparticle dimensions of $190 \mathrm{~nm}$ diameter and $25 \mathrm{~nm}$ height (the particles of this size are structurally very stable and exhibit a well manifested plasmon-related light-absorption peak) according to the Hole-Mask Colloidal Lithography method. Subsequent depositions of the metal constituents matching the targeted composition, are followed by high-temperature annealing to induce the alloy formation. ${ }^{21}$ This approach yields homogeneously alloyed polycrystalline disk-shaped nanoparticles ${ }^{4,21}$ with narrow size distribution (Figs. 1b and S1-4).

As the first polymer coating we chose polytetrafluoroethylene (PTFE) owing to its high chemical resistance and hydrophobicity. RF-sputtering of a $30 \mathrm{~nm}$ thin layer from a PTFE target yields a conformal coating of the entire nanoparticle array (Figs. 1c and S7-8). The optical response of the $\mathrm{Pd}_{70} \mathrm{Au}_{30}$ and $\mathrm{Pd}$ systems, measured in transmission configuration, is shown before and after applying the PTFE coating (Fig. 1d-e). The corresponding optical extinction spectra feature a localized surface plasmon resonance (LSPR) peak, which broadens and shifts to the red after PTFE deposition, due to the high refractive index of the coating. ${ }^{23}$ Upon exposure to $1000 \mathrm{mbar}$ of hydrogen, this peak further broadens and red-shifts owing to the change in both volume and permittivity of the metal nanoparticles, induced by hydrogen population of the interstitial lattice sites. ${ }^{15}$ This LSPR peak shift, which is a linear function of the hydrogen concentration inside the nanoparticles, ${ }^{22}$ then serves as the basis for our nanoparticle-polymer hybrid optical hydrogen sensors. 

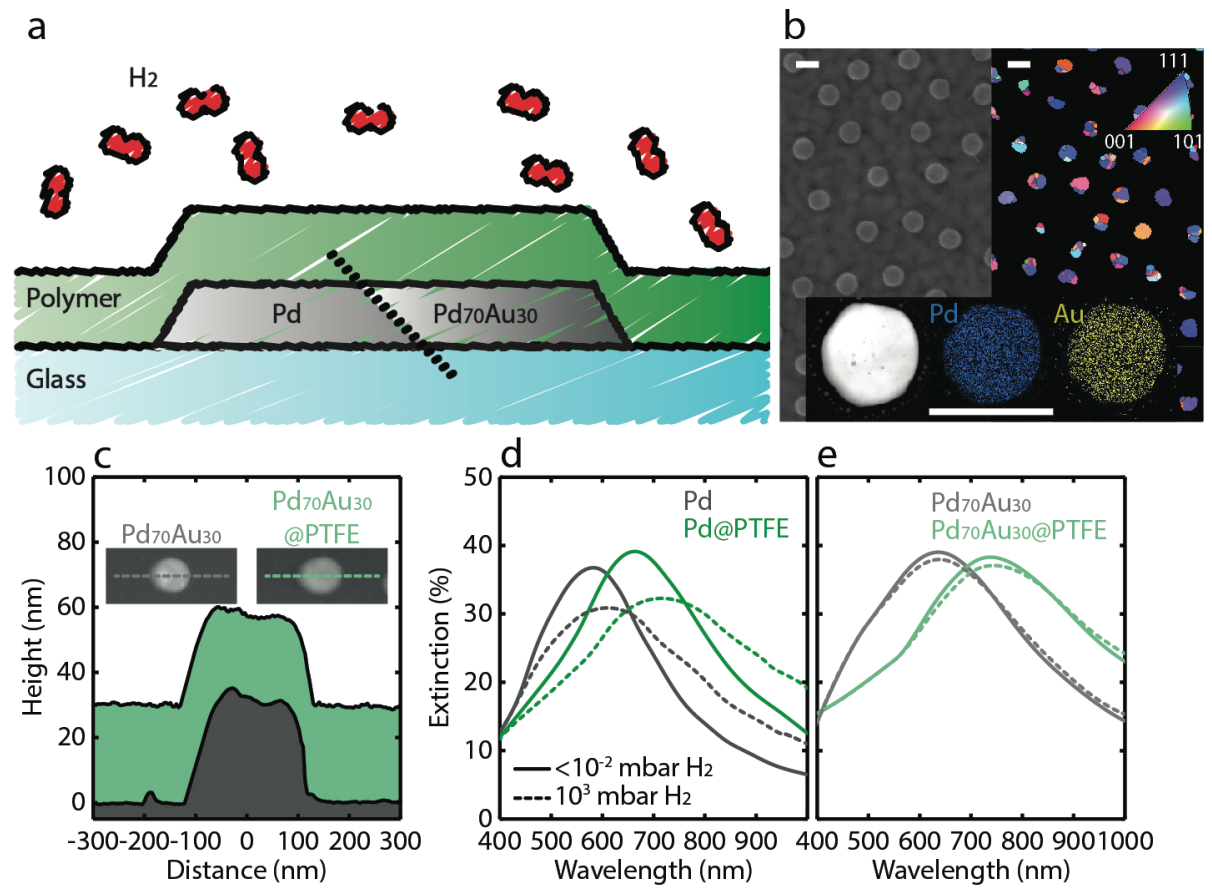

Figure 1. Plasmonic metal-polymer hybrid nanomaterial architecture and characterization.

(a) Artist's rendition of a plasmonic metal-polymer nanomaterial comprising hydride-forming Pd or $\mathrm{Pd}_{70} \mathrm{Au}_{30}$ alloy nanoparticles, and a thin polymer coating. (b) SEM image (left) and Transmission Kikuchi Diffraction (TKD) micrographs (right) of a $P d_{70} A u_{30}$ nanoparticle array, and EDX elemental maps of an individual $P d_{70} A u_{30}$ alloy nanoparticle (bottom). The color code of the TKD images is explained in the inverse pole figure plot, which depicts the bulk grain crystallographic orientation with respect to the out-of-plane axis. Scale bars are $200 \mathrm{~nm}$. (c) AFM image of an individual $P d_{70} A u_{30}$ alloy particle before and after PTFE coating. The profile of the coated particle is shifted upward by the nominal $30 \mathrm{~nm}$ PTFE thickness for clarity. Optical extinction spectra of $a(d) P d$ and (e) $P d_{70} A u_{30}$ alloy nanoparticle array before and after PTFE

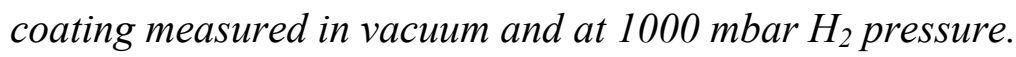

\section{Sensor@PTFE: accuracy and limit of detection}

As the first characterization of our sensors, we measured optical hydrogen absorption and desorption isotherms at $30{ }^{\circ} \mathrm{C}$ for pure $\mathrm{Pd}$ and the $\mathrm{Pd}_{70} \mathrm{Au}_{30}$ alloy in the absence and presence of the $30 \mathrm{~nm}$ PTFE coating (Fig. 2a-b). For both systems the optical readout, i.e. the spectral shift of the plasmonic peak, $\Delta \lambda_{\text {peak }}$, is proportional to the $H$ concentration in the host, expressed as 
H/Pd ratio. ${ }^{15,22}$ For Pd, a characteristic $\alpha$-phase region at low hydrogen partial pressure, where hydrogen is diluted at low concentration in a solid solution, is observed. At a critical hydrogen pressure, the $\alpha+\beta$ phase coexistence region ("plateau") at the first-order phase transition to and from the hydride ( $\beta$-phase) appears and exhibits wide hysteresis. Finally, the pure $\beta$-phase region at high hydrogen partial pressure is observed. ${ }^{24}$ In contrast, the $\mathrm{Pd}_{70} \mathrm{Au}_{30}$ system exhibits a monotonous hysteresis-free response. Interestingly, adding the PTFE coating to the two sensors not only retains the identified beneficial properties of the $\mathrm{Pd}_{70} \mathrm{Au}_{30}$ alloy but also enhances the signal amplitude by approximately a factor of two throughout the entire pressure range investigated. This is remarkable because, as becomes clear from quartz-crystal microbalance (QCM) measurements, this enhancement is not caused by hydrogen absorption in the PTFE itself (Fig. 2c-d). Specifically, our analysis indicates that the same amount of hydrogen is absorbed with and without PTFE coating both for Pd and the $\mathrm{Pd}_{70} \mathrm{Au}_{30}$ (the absolute numbers expressed as $\mathrm{H} / \mathrm{Pd}$ ratio are in good agreement with earlier reports ${ }^{20,25}$ ), corroborating that hydrogen only interacts with the nanoparticles. Furthermore, our Density Functional Theory (DFT) calculations show that the interaction between $\mathrm{H}_{2}$ and PTFE (and PMMA) consists of only weak dispersion forces (Fig. S11).

This contrasts earlier work reporting on nearly doubled hydrogen solubility in MOF-coated Pd nanoparticles. ${ }^{26}$ To address this aspect in more detail, we note that the sensitivity of a plasmonic hydrogen sensor is dictated by the spectral position of the plasmonic resonance, $\lambda_{\text {peak }}$, in the nonhydrogenated state. ${ }^{22}$ Since adding a polymer coating spectrally red-shifts $\lambda_{\text {peak }}$ (cf. Fig. 1d-e), we expect an increase in sensitivity. This is indeed the case and the reason for the observed enhancement of the signal amplitude, as we discuss in detail in Supplementary Information based on Finite-Difference Time-Domain (FDTD) simulations. Since this enhancement is a purely optical far-field effect (the concentration of absorbed hydrogen remains the same), it is generic to any (polymer) coating and can be maximized by a high refractive index of the latter, as well as by increasing its thickness (Figs. S16-17) at the cost of a slight reduction of the figure-of-merit of the plasmonic sensor readout (Fig. S18). At the same time, it is exclusive to plasmonic hydrogen sensors based on nanoparticles since no such optical signal enhancement was observed for PTFE-coated Pd thin films. ${ }^{27}$ Finally, we also note that the observed symmetric lowering of the plateau pressures for the Pd@PTFE system (cf. Fig. 2a and c) hints at strain imposed by the PTFE layer ${ }^{28}$ (for the related estimates, see Supplementary Information) 
The limit of detection ( $\mathrm{LoD}$, the lowest change in concentration of analyte that can be distinguished by a sensor) of the Pd@PTFE and $\mathrm{Pd}_{70} \mathrm{Au}_{30} @$ PTFE sensors is derived by exposing them to pulses of gradually decreasing $\mathrm{H}_{2}$ pressure in vacuum (from 1000 to $7 \mu$ bar - the lowest pressure attainable in our setup) and by measuring the spectral shift of the plasmonic resonance peak maximum, $\Delta \lambda_{\text {peak }}$, at $1 \mathrm{~Hz}$ sampling frequency (Fig. 2e). Defining the $\mathrm{LoD}$ as $3 \sigma$, where $\sigma$ is the noise of the acquired signal (i.e. $0.01 \mathrm{~nm}$, see corresponding discussion in Supplementary Information and Fig. S20), we extrapolate that $\mathrm{LoD}<5 \mu$ bar in pure hydrogen (Fig. 2f). To assess the sensitivity under more realistic conditions, we also conducted similar experiments for the $\mathrm{Pd}_{70} \mathrm{Au}_{30} @$ PTFE sensor in $\mathrm{Ar}$ and in synthetic air carrier gas at atmospheric pressure in a flow reactor setup. In both backgrounds, down to the lowest $\mathrm{H}_{2}$ concentration experimentally attainable, i.e. $10 \mathrm{ppm}$ and $20 \mathrm{ppm}$, respectively, a $\Delta \lambda_{\text {peak }}$ response is clearly discernible. This places our system amongst the most sensitive hydrogen sensors reported to-date under simulated application conditions in air, ${ }^{29,30}$ notably with significant potential to further enhance the sensitivity by e.g. tailoring the coating layer material and its thickness, and by optimizing nanoparticle dimensions. ${ }^{22}$ To this end, extrapolating the LoD based on the experimentally derived noise in flow conditions (Fig. S24), we derive an LoD of 1 ppm in Ar and 5 ppm in synthetic air.
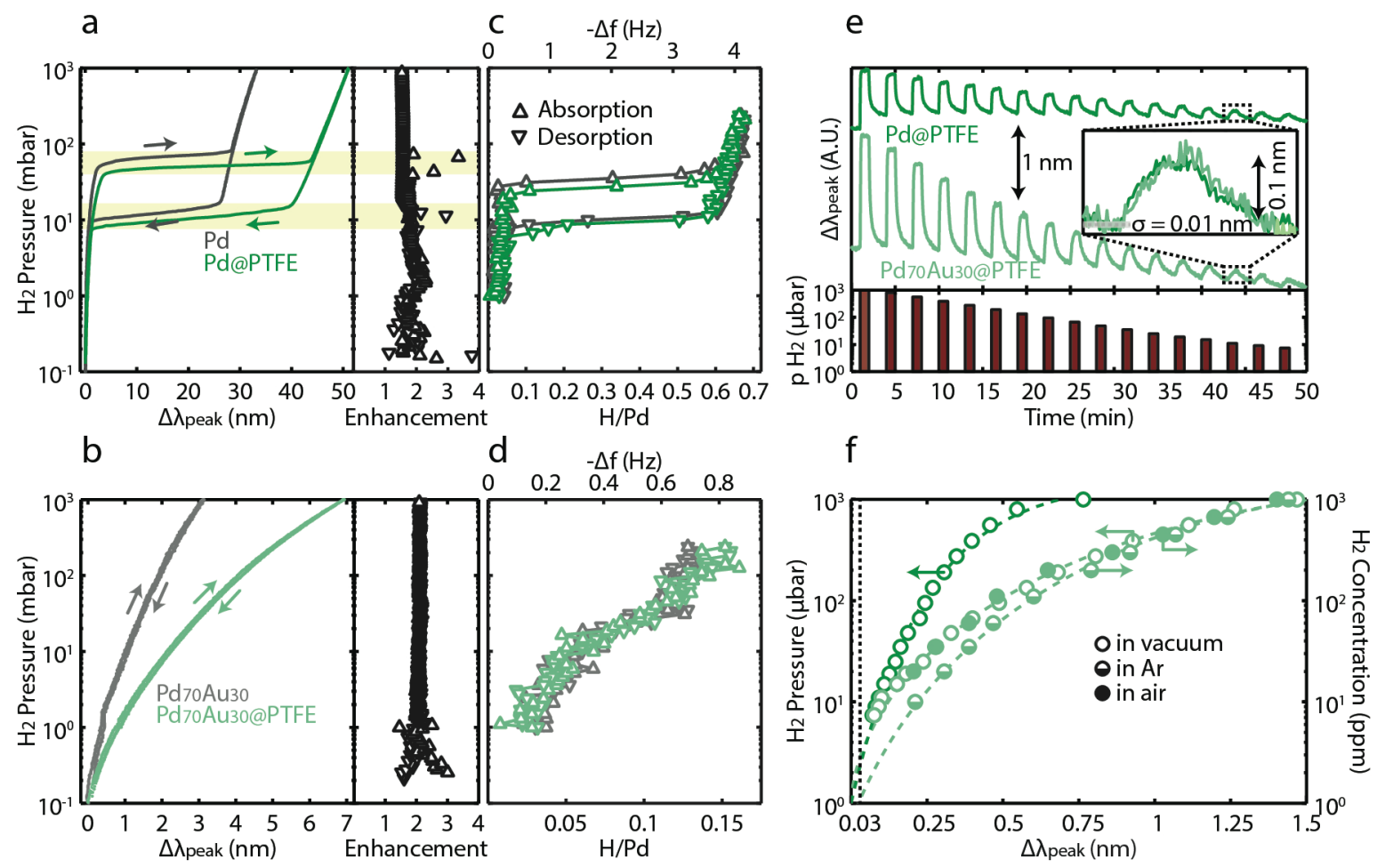


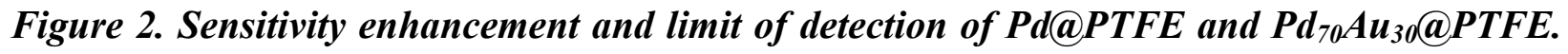
Optical absorption and desorption isotherms of (a) $P d$ and (b) $P d_{70} A u_{30}$ alloy sensors before and after PTFE coating. The arrows denote the sorption direction. The panels to the right depict the $\Delta \lambda_{\text {peak }}$ ratio of the coated and uncoated sensors, and reveal a factor of two enhanced signal amplitude induced by the PTFE. Isotherms of (c) Pd and (d) Pd $d_{70} A u_{30}$ sensors before and after PTFE coating measured by quartz-crystal microbalance in a flow chamber at ambient pressure, using Ar as carrier gas. They reveal that the hydrogen concentration in the metal, expressed as $H / P d$, is in excellent agreement with previous reports, ${ }^{20}$ and that no $\mathrm{H}_{2}$ is absorbed in the PTFE itself due to the identical response by the coated and uncoated system. (e) $\Delta \lambda_{\text {peak }}$ response to a step-wise decreasing $\mathrm{H}_{2}$ pressure in the 7-1000 $\mu$ bar range, measured at $1 \mathrm{~Hz}$ sampling frequency in a vacuum chamber. The inset shows the data at $10 \mu$ bar $\mathrm{H}_{2}$ to define the noise, $\sigma$, as $0.01 \mathrm{~nm}$ (also see corresponding discussion in Supplementary Information). (f) Measured $\Delta \lambda_{\text {peak }}$ as a function of the $\mathrm{H}_{2}$ pressure derived from (e) and also from measurements in Ar and synthetic air background down to the lowest attainable hydrogen partial pressure in our system, 10 ppm and 20 ppm, respectively (Figs. S25-26). The green lines depict an extrapolation from the lowest reliably attainable data point in our system for the Ar and air background to the $3 \sigma$ point, indicating a LoD $<1$ ppm and 5 ppm, respectively. The black dashed line marks the extrapolated LoD at $3 \sigma=0.03 \mathrm{~nm}$. All experiments were done at $30^{\circ} \mathrm{C}$.

\section{Sensor@PTFE: response time}

As the next characterization step, we measured the response times of uncoated and PTFE-coated $\mathrm{Pd}$ and $\mathrm{Pd}_{70} \mathrm{Au}_{30}$ alloy sensors at $30{ }^{\circ} \mathrm{C}$, by monitoring their temporal response to a step-wise in/decrease of hydrogen pressure to/from $40 \mathrm{mbar}_{2}$ (i.e. the lower flammability limit) in a vacuum chamber (Figs. 3a-b and S28 for recovery times). We observe two key effects, namely that (i) for both ab- and desorption the response time of the uncoated $\mathrm{Pd}_{70} \mathrm{Au}_{30}$ sensor is significantly shorter than for the uncoated Pd one, and (ii) the PTFE coating for both systems further shortens the response time to significantly below one second for the alloy. The detailed quantitative analysis of the $\mathrm{Pd}$ and $\mathrm{Pd}_{70} \mathrm{Au}_{30}$ ab- and desorption kinetics via Arrhenius analysis (Figs. S29-30) reveals a significant reduction of the apparent activation energies, $E_{a}$, of the ratelimiting steps for both hydrogen absorption and desorption. This is due to the PTFE coating 
(Figs. 3d-e and S29-30), which also refines the understanding of the observed hydrogen sorption kinetics acceleration in PTFE-coated Pd thin films reported by Ngene et al. ${ }^{27}$ These results, along with similar kinetics acceleration observed for MOF-coated $\mathrm{Pd}$ sensors measured in vacuum/hydrogen ${ }^{26}$ and air, ${ }^{31}$ imply that the kinetics-accelerating effect of such coatings may be generic. To this end, a number of different explanations to the origin of the accelerated kinetics is given in the literature, such as the modification of surface chemical ${ }^{27,32}$ and electronic states $^{26,27,33}$, physical force/stress imposed by the coating layer ${ }^{33}$ and the removal of competing molecules reacting on the surface. ${ }^{31}$

Employing DFT calculations to capture the experimentally observed trends, we show that the measured decrease in $E_{a}$ induced by the polymer coating is connected to the absorption and desorption processes at the nanoparticle-polymer interface (Fig. 3e), mediated by polymer-metal bond formation verified by XPS analysis (Fig. S9). On one hand, the activation barrier for hydrogen absorption from surface to subsurface sites is reduced by $11 \mathrm{~kJ} / \mathrm{mol}$ upon coating by PTFE, resulting in faster absorption. On the other hand, for sites close to PTFE, surface-adsorbed hydrogen is destabilized by $9 \mathrm{~kJ} / \mathrm{mol}$, which results in faster $\mathrm{H}_{2}$ desorption during dehydrogenation. In other words, these effects give rise to a reduction of $E_{a}$ for both hydrogen absorption and desorption, explaining the experimentally observed accelerated kinetics and measured lower apparent activation barriers for the respective rate-limiting step. Concerning the kinetics, we also note that the dependence of the response time on pressure (Fig. 3g and Figs. 4e and $\mathbf{5 c}$ below) is fairly weak and close to the power-law form with a small exponent (cf. Eq. S15). This kinetic feature is indicative of energetic heterogeneity at the surface of the alloy (see corresponding discussion in Supplementary Information).

Having established that the hydrogen absorption rate into the bulk of the nanoparticle sensors is limited by their surface, ${ }^{34}$ the time scale for this process is expected to be proportional to their volume/surface ratio, with the proportionality constant depending on the specifics of the kinetics at the surface (Figs. S35 and S37 and the corresponding discussion). Hence, as a key advantage enabled by a sensor platform based on nanoparticles, tailoring the volume/surface ratio should render a faster response. Indeed, reducing the mean diameter of the nanoparticles in a $\mathrm{Pd}_{70} \mathrm{Au}_{30} @$ PTFE sensor from $190 \mathrm{~nm}$ to $100 \mathrm{~nm}$ further reduces the response time for desorption from ca. $9 \mathrm{~s}$ to $3 \mathrm{~s}$ (Fig. S28), and for absorption to (below) the resolution limit of our system at $0.3 \mathrm{~s}$ (Figs. 3c). Hence, the $100 \times 25 \mathrm{~nm} \mathrm{Pd}_{70} \mathrm{Au}_{30} @$ PTFE sensor meets the toughest of the US 
DoE targets, ${ }^{2}$ defined as response time of $<1 \mathrm{~s}$ to $1 \mathrm{mbar}$ hydrogen at ambient temperature (Fig. 3f-g). Furthermore, a recovery time of below $5 \mathrm{~s}$ throughout the same hydrogen pressure range is also achieved (Fig. S32), representing the current state-of-the-art in this respect. To this end, we highlight that additional downsizing and volume/surface engineering of the nanostructures offers potential to further improve these already very impressive response times, in particular when using optimized nanoparticle designs, such as nanorods that combine a spectrally red-shifted LSPR for optimized LoD with small volume/surface, are used. However, on the note of utilizing the PTFE coating as selective membrane to prevent sensor deactivation by trace gases like $\mathrm{CO}$ and $\mathrm{NO}_{2}$ present in air, we find that no sufficient protection is obtained. This is evidenced by the deactivation tests performed in a flow reactor operating at atmospheric pressure, using synthetic air as the carrier gas to mimic real application conditions. In particular, the sensor was exposed to 10 cycles of $4 \% \mathrm{H}_{2}$ followed by 10 cycles of $4 \% \mathrm{H}_{2}$ plus an interfering gas, i.e. $3 \% \mathrm{CO}_{2}, 0.5 \%$ $\mathrm{CH}_{4}, 0.1 \% \mathrm{CO}, 0.05 \% \mathrm{NO}_{2}$ (Fig. S49).
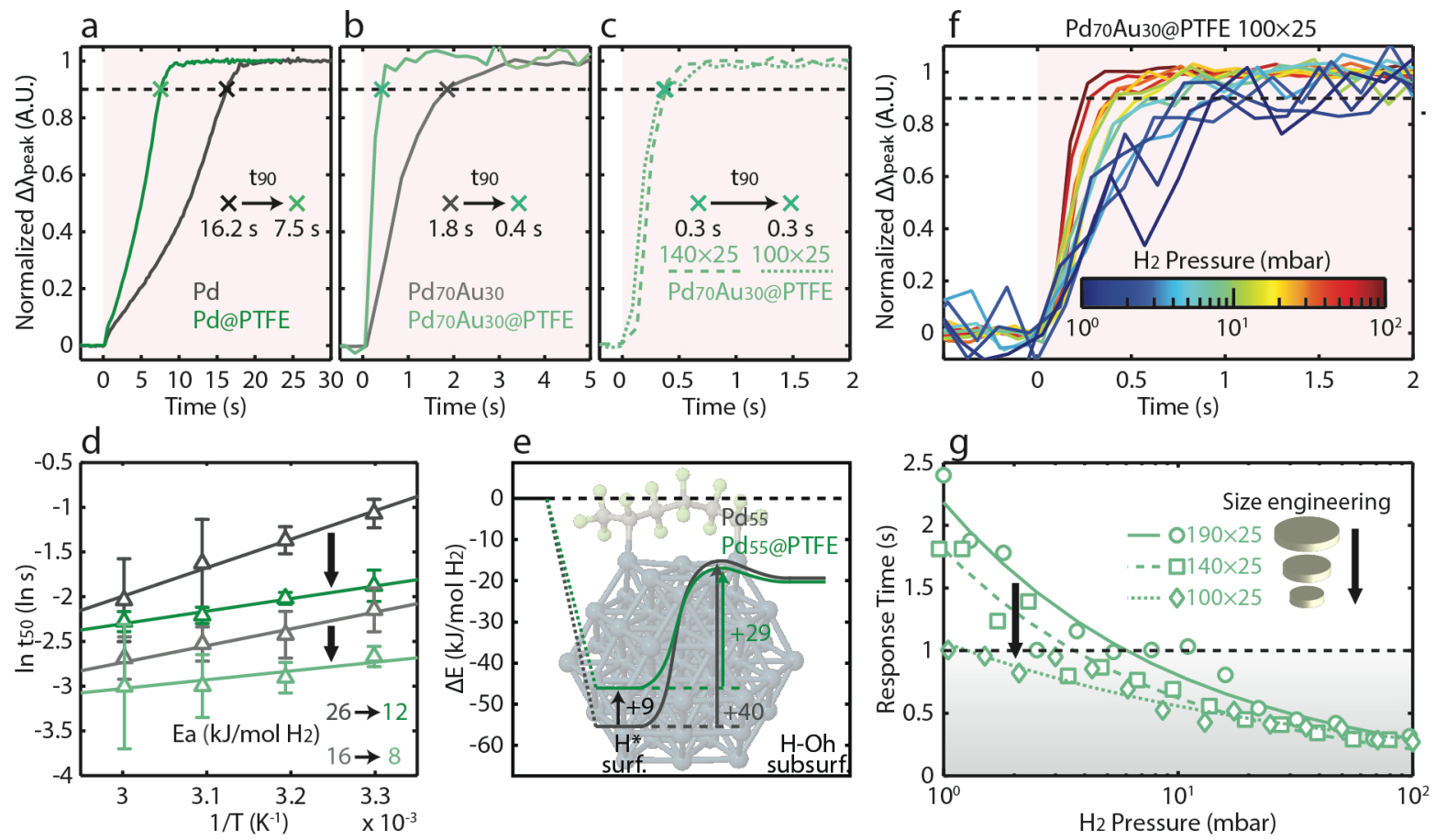

Figure 3. Response times of PTFE-coated Pd and Pd $\mathrm{P}_{70} \mathrm{Au}_{30}$ at room temperature. Accelerated absorption kinetics are observed for PTFE-coated (a) Pd and (b) Pd $d_{70} A u_{30}$ sensors with $190 \times 25$ nm average nanoparticle dimensions (first and second number denotes diameter and height, respectively). $t_{90}$ is the response time defined as the time to reach $90 \%$ of the signal and is marked by black dashed lines. (c) Engineering the volume/surface ratio by employing 
nanoparticles with smaller dimensions enables even faster absorption kinetics, as shown for 140 $\times 25$ and 100 $\times 25$ Pd $d_{70} A u_{30} @ P T F E$ sensors. The shaded areas in panels (a-c) mark the period when the sensors are exposed to 40 mbar $H_{2}$. (d) Experimentally measured apparent hydrogen absorption activation energies, $E_{a}$, of a $P d$ and $P d_{70} A u_{30}$ sensor before and after PTFE coating, revealing a distinct reduction of $E_{a}$ upon PTFE coating. The error bars are the standard deviation from three measurements. (e) Energy landscape for hydrogen absorption in $P d_{55}$ with and without PTFE adsorbed on the surface calculated by DFT. The figure depicts the states of adsorbed $H$ on the surface ( $H^{*}$ surf.) and absorbed $H$ in a subsurface octahedral site (H-Oh subsurf.). The reference is taken as $P d_{55}+1 / 2 H_{2}$ (g) and $P d_{55} @ P T F E+1 / 2 H_{2}(g)$, respectively. The background image depicts the modelled Pd55@PTFE system. (f) $100 \times 25$ nm Pd70Au 30 PTFE sensor response to $H_{2}$ pressure pulses in the range from 1000 to $1 \mathrm{mbar}$. (g) Response time of $P d_{70} A u_{30} @ P T F E$ sensors with different sizes to varying $\mathrm{H}_{2}$ pressure pulses. For the $100 \times 25 \mathrm{~nm}$ sensor, sub-second response is achieved throughout the investigated pressure range down to 1 mbar, making this the fastest hydrogen sensor ever reported at these conditions (Table S2). The lines denote a power-law fit based on Eq. (S18).

\section{$\mathbf{P d}_{70} \mathrm{Au}_{30} @$ PMMA Sensor}

As a response to the shortcomings of the PTFE coating, in the second part of our study we investigate an alternative polymer system - poly(methyl methacrylate), PMMA, for which excellent $\mathrm{H}_{2}$ selectivity to other gases has been demonstrated. ${ }^{35-37}$ To assess the molecular sieving function of a PMMA coating, we spin coated a $35 \mathrm{~nm}$ thick PMMA film onto a $190 \times 25$ $\mathrm{Pd}_{70} \mathrm{Au}_{30}$ sensor (for a neat $\mathrm{Pd} @ \mathrm{PMMA}$ analogue, see Supplementary Information). The corresponding $\mathrm{Pd}_{70} \mathrm{Au}_{30} @$ PMMA sensor response in an identical deactivation test as described above for the PTFE sensor is summarized in Fig. 4a, revealing the excellent protection provided by the PMMA layer. Specifically, as depicted in Fig. 4b, the sensor signal for all interfering gases is with significant margin retained within the $\pm 20 \%$ deviation limit from the normalized $\Delta \lambda_{\text {peak }}$ in pure $4 \% \mathrm{H}_{2}$ according to the performance standard for hydrogen sensors. ${ }^{38}$ This is remarkable in view of the strong deactivation of the uncoated control (Fig. S49) by, for example, $\mathrm{CO}$ through the effective poisoning of the surface via strong $\mathrm{CO}$ chemisorption and the concurrent blocking of hydrogen dissociation sites. ${ }^{18}$ 

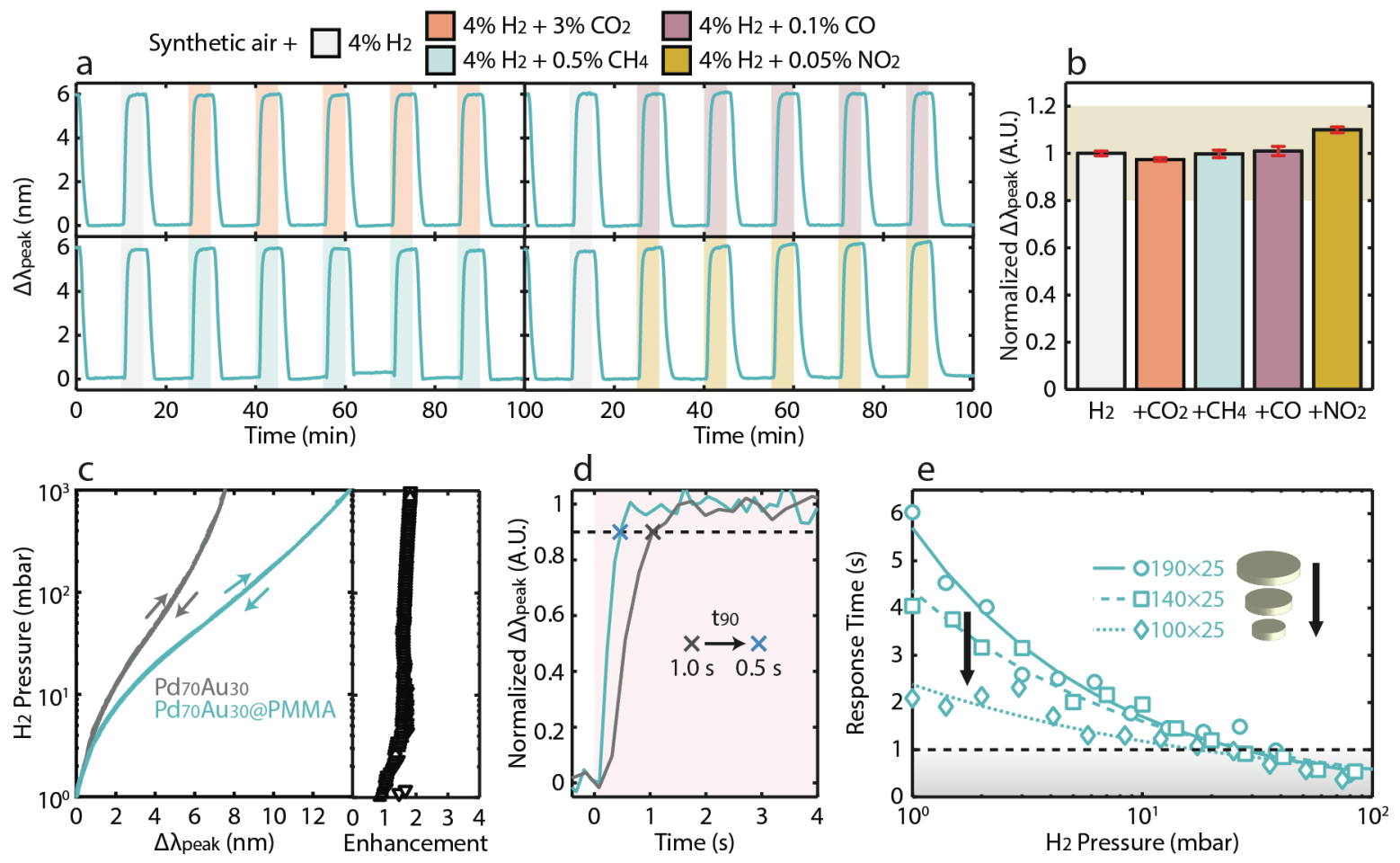

Figure 4.Pd70 Au $30 @ P M M A$ Sensor. (a) Time-resolved $\Delta_{\text {peak }}$ response of $P d_{70} A u_{30} @ P M M A$ to a pulse of $4 \% \mathrm{H}_{2}$ followed by 5 pulses of, respectively, $4 \% \mathrm{H}_{2}$ and $3 \% \mathrm{CO}_{2}, 4 \% \mathrm{H}_{2}$ and $0.5 \%$ $\mathrm{CH}_{4}, 4 \% \mathrm{H}_{2}$ and $0.1 \% \mathrm{CO}$, and $4 \% \mathrm{H}_{2}$ and $0.05 \% \mathrm{NO}_{2}$ in synthetic air carrier gas, measured at atmospheric pressure. The $\Delta \lambda_{\text {peak }}$ signals are retained throughout exposure to the different background gases, as summarized in (b) where the respective signals are normalized to the one obtained in pure $4 \% \mathrm{H}_{2}$. The error bars denote the standard deviation from 10 cycles. The shaded area indicates the $\pm 20 \%$ deviation limit from the normalized $\Delta \lambda_{\text {peak }}$ in pure $4 \% H_{2}$. (c) Optical absorption and desorption isotherms of the $P d_{70} A u_{30}$ sensor measured before and after adding of the PMMA coating. The arrows denote the sorption direction. The panel to the right depicts the $\Delta \lambda_{\text {peak }}$ ratio of the coated and uncoated sensors, revealing a similar signal enhancement factor to the one found for the PTFE-coated system. (d) Pd $d_{70} A u_{30}$ sensor response before and after PMMA coating to a step-wise $\mathrm{H}_{2}$ pressure in/decrease to/from 40 mbar. (e) Response times of $P d_{70} A u_{30} @ P M M A$ sensors with different nanoparticle sizes to different $\mathrm{H}_{2}$ pressure pulses. The lines denote a power-law fit based on Eq. (S18). All experiments were done at $30^{\circ} \mathrm{C}$. 
Now, referring to the beneficial effects of the PTFE coating on the sensor performance identified above, we find that the same signal amplitude enhancement by approximately a factor of two is also observed for PMMA (Fig. 4c). Hence, as anticipated and discussed above, this is indeed a generic effect inherent to dielectric (polymer) coatings. The observed similar magnitude of the enhancement for PTFE and PMMA is thus solely the consequence of their similar refractive indices and the essentially identical thickness of the coatings, since also for PMMA no relevant hydrogen sorption occurs within the polymer itself (Fig. S11). In terms of response time shortening, our analysis reveals that this effect is also present for a PMMA coating, as the response time at $30{ }^{\circ} \mathrm{C}$ to a step-wise increase of the hydrogen pressure to $40 \mathrm{mbar}_{2} \mathrm{H}_{2}$ for the $\mathrm{Pd}_{70} \mathrm{Au}_{30} @$ PMMA sensor is reduced to $0.5 \mathrm{~s}$ (Fig. 4d). However, as more detailed analysis reveals, the acceleration of the hydrogen sorption kinetics is on average a factor two smaller for PMMA compared to PTFE and thus, even by reducing the nanoparticle diameter to $100 \mathrm{~nm}$, the system falls slightly short of the DoE target for sub-second response to 1 mbar at room temperature (Fig. 4e). The reason we find is the smaller reduction of the apparent activation energy of the rate-limiting steps during hydrogen absorption and desorption induced by PMMA, compared to PTFE (Figs. S42-43), which is also confirmed by DFT calculations (Fig. S15).

\section{$\mathbf{P d}_{70} \mathrm{Au}_{30} @$ PTFE/PMMA Tandem Sensor}

As an intermediate conclusion it becomes clear that the ideal polymer layer essentially would combine the properties of the tested PTFE and PMMA systems. Hence, we fabricated a $\operatorname{Pd}_{70} \mathrm{Au}_{30}$ hydrogen sensor encapsulated by a tandem $30 \mathrm{~nm}$ PTFE + $35 \mathrm{~nm}$ PMMA structure. In this way, we are able to test the hypotheses that (i) it indeed is the PTFE-nanoparticle interface and the corresponding reduction of the apparent activation barriers that gives rise to the superior response time, (ii) PMMA functions as a superior molecular sieve layer, and (iii) these two functions can be combined in a heterostructure. To prepare the sensor, we applied a $5 \mathrm{~s} \mathrm{H}_{2}$ plasma treatment to the previously already tested $\mathrm{Pd}_{70} \mathrm{Au}_{30} @$ PTFE sensor (cf. Figs. 1-3) to render the PTFE-surface hydrophilic. ${ }^{39}$ This enables wetting and thus spincoating of PMMA dissolved in methoxybenzene solvent. The obtained polymer tandem structure is schematically depicted in Fig. 5a, together with a cross-section SEM micrograph in which a PdAu alloy nanoparticle encapsulated in the tandem polymer arrangement is clearly resolved. The effective coating of the PTFE layer by the PMMA is further confirmed by XPS analysis (Fig. 5b). 

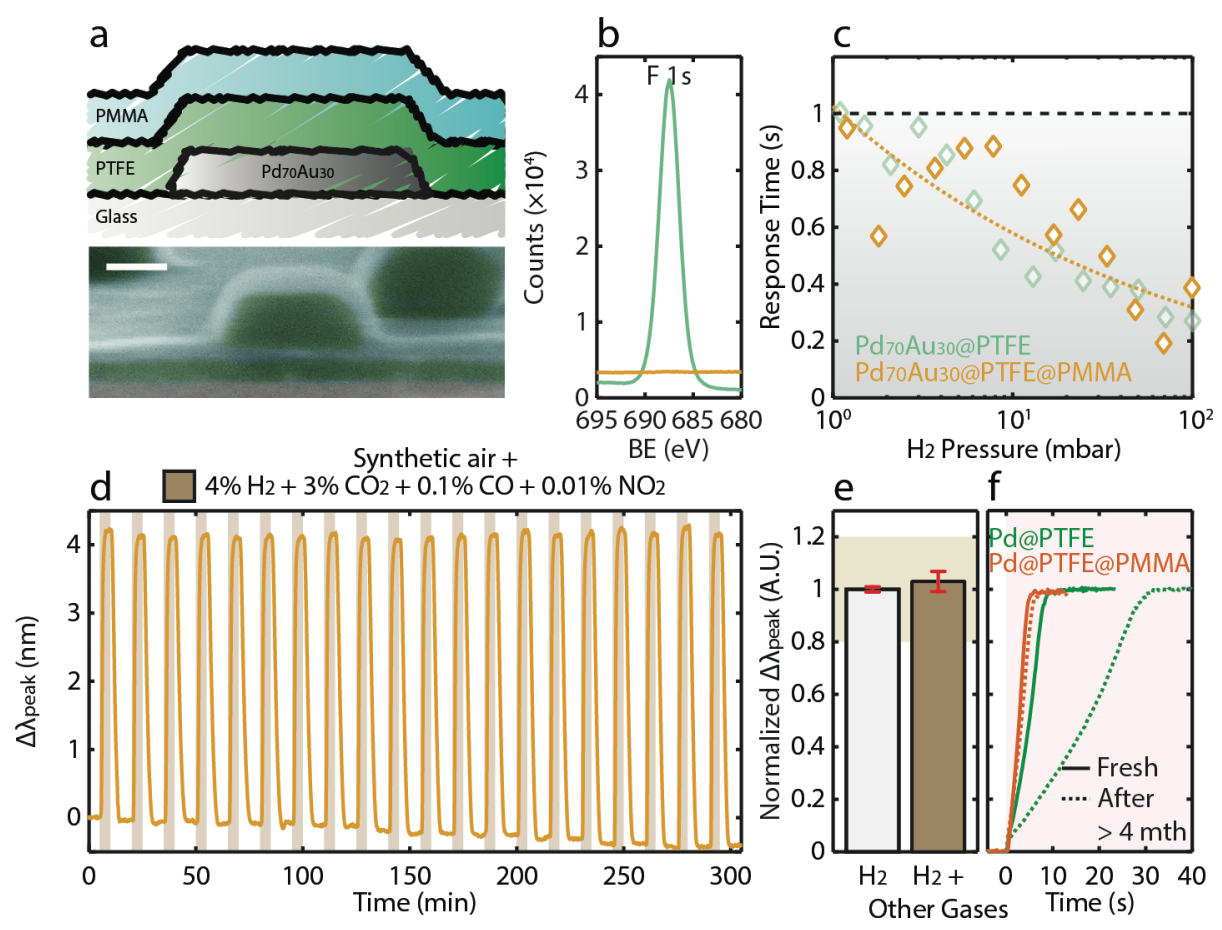

Figure 5.Pd70Au30@PTFE@PMMA Tandem Sensor. (a) Top: Artist's rendition of a tandem sensor comprising a heterostructure coating of PTFE and PMMA layers on top of a Pd $d_{70} A u_{30}$ alloy nanoparticle array. Bottom: Cross-section SEM image of the tandem sensor revealing the polymer layers with different contrast. Scale bar is $100 \mathrm{~nm}$. (b) XPS spectra of the F 1s peak of a

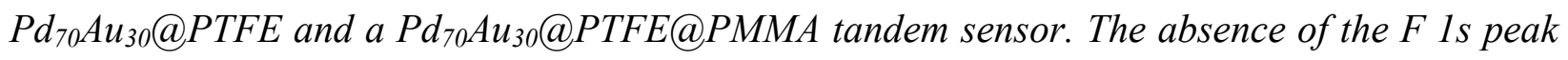
for the tandem confirms the effective coating of the PTFE by PMMA. (c) Response times of a tandem sensor with $100 \times 25 \mathrm{~nm}$ nanoparticle dimensions to different $\mathrm{H}_{2}$ pressure pulses, plotted together with the ones of the same sensor with only the PTFE coating. The dashed-line denotes a power-law fit based on Eq. (S18). (d) $\Delta \lambda_{\text {peak }}$ of the tandem sensor for 20 cycles of $4 \% \mathrm{H}_{2}$ plus $3 \%$ $\mathrm{CO}_{2}, 0.1 \% \mathrm{CO}$ and $0.05 \% \mathrm{NO}_{2}$ in synthetic air. (e) $\Delta \lambda_{\text {peak }}$ in poisoning gas background normalized to $\Delta \lambda_{\text {peak }}$ in pure $4 \% \mathrm{H}_{2}$ revealing the excellent deactivation resistance of the tandem. The error bars denote the standard deviation from 20 cycles. The shaded area indicates the $\pm 20 \%$ deviation limit from the normalized $\Delta \lambda_{\text {peak }}$ in pure $4 \% \mathrm{H}_{2}$. (f) Absorption kinetics of Pd@PTFE and Pd@PTFE@PMMA sensors to 40 mbar $\mathrm{H}_{2}$ at different aging states. After four months in ambient conditions, the tandem sensor retains its kinetic properties while the PTFEonly-coated sensor has significantly deteriorated. 
Assessing the room temperature response time in the same way as above reveals that for the tandem coating it is essentially identical to the PTFE coating alone (Fig. 5c). Since we use the same sensor in both cases also the reproducibility as such is remarkable. Furthermore, and as the key result, the sub-second response is again retained down to 1 mbar, meeting the DoE target. Comparing the apparent activation energies for the two systems, we find them to be identical (Fig. S46), corroborating that the kinetics acceleration observed indeed is governed solely by the direct interaction between coating material and nanoparticle surface. At the same time, the deactivation tests in synthetic air, following the same experimental scheme used above, reveal that the same deactivation resistance as for the PMMA alone is achieved (Fig. S49), convincingly confirming that the hybrid material concept is able to combine multiple functionalities of its constituents in a single system. Moreover, in a significantly exaggerated test, in terms of concentrations to "mimic" the environment of an urban area, the sensor with the tandem coating shows no sign of deactivation when exposed to twenty $4 \% \mathrm{H}_{2}$ pulses mixed with $3 \% \mathrm{CO}_{2}, 0.1 \% \mathrm{CO}$ and $0.01 \% \mathrm{NO}_{2}$ (Fig. 5d). Also in this tough test, the absolute response remains well within the $\pm 20 \%$ deviation limit according to the performance standard for hydrogen sensors ${ }^{38}$ (Fig. 5e). This excellent deactivation resistance is also retained in the long term. In particular, after four-month exposure to ambient conditions, the tandem sensor retains its response, while the only PTFE-coated sensor is significantly deactivated as indicated by drastically reduced response time (Fig. 5f). Finally, as an additional advantage of the tandem system, owing to its doubled polymer layer thickness, the sensitivity is further enhanced by $30 \%$ compared to the PTFE layer alone (Figs. S47-48), in agreement with our FDTD simulations (Fig. S17).

In summary, the concerted hydrogen sorption rate and sensitivity enhancement, as well as molecular sieving effects, we find for polymer coating layers on hydride-forming metal nanoparticles provide a new mechanism by which plasmonic nanoparticle arrays can operate as optical hydrogen sensors with unprecedented response metrics. The performance of the plasmonic metal-polymer optical hydrogen sensors presented here challenges existing hydrogensensing technologies (Table S2). Specifically, they (i) feature sub 5-ppm detection limit, (ii) exhibit deactivation resistance by poisoning gases such as $\mathrm{O}_{2}, \mathrm{CO}_{2}, \mathrm{CH}_{4}, \mathrm{NO}_{2}$ and $\mathrm{CO}$, (iii) feature remote optical readout without risk for spark generation, (iv) are hysteresis-free, (v) exhibit excellent long-term stability, and (vi) meet for the first time the stringent $1 \mathrm{~s}$ response 
time target at ambient temperature and $1 \mathrm{mbar}_{2}$ partial pressure. The last point is achieved by concerting two key effects: (i) reducing the activation barrier for surface-to-subsurface hydrogen diffusion via polymer-metal surface bond formation; and (ii) tailoring the volume/surface ratio, a feature uniquely possible in nanoparticles, to overcome the intrinsic response time limitations imposed by the identified surface-associated rate-limiting steps during (de)hydrogenation of the sensor. The latter point also implies that it is fundamentally impossible to reach the $1 \mathrm{~s}$ response time target at low hydrogen partial pressures with macroscopic (bulk) or, to some extent, thinfilm systems (see corresponding discussion in Supplementary Information) based on Pd and its alloys, since in this regime the volume/surface ratio is the critical factor determining the response time. In a wider perspective, our work opens the door to next-generation (optical) gas sensors centered on the idea of a hybrid material that combines tailored plasmonic signal transducers with multiple selective membrane materials which, by engineering the dimensions and interfaces of the material constituents, enable the optimization of sensitivity, selectivity, deactivation resistance and response times, to meet the ever increasing demand for advanced sensor technologies.

\section{Methods}

Sensor fabrication. Evaporation masks were fabricated using the standard hole-mask colloidal Lithography (HCL) process ${ }^{40}$ on $1 \times 1 \mathrm{~cm}^{2}$ glass substrates (Borofloat, Schott Scandinavia AB), on TEM "windows" made in-house following the procedure by Grant et al., ${ }^{41}$ on silicon wafer substrates and on QCM crystals (Laptech, SC-cut, $10 \mathrm{MHz}$ fundamental frequency) pre-coated with $100 \mathrm{~nm} \mathrm{CVD-grown} \mathrm{SiO}_{2}$ (PECVD, STS) on one of the Au electrodes. The steps of the mask fabrication were as follows: (i) substrates were cleaned (by ultrasonic agitation for the case of glass, silicon and QCM crystals and only by rinsing for the TEM windows in order to not break the membrane) subsequently in acetone, isopropanol (IPA), and de-ionized (DI) water. (ii) Poly(methyl methacrylate) (PMMA, MicroChem Corporation, 4 wt. $\%$ diluted in anisole, $\mathrm{M}_{\mathrm{W}}=$ 950 000) was spin-coated onto the substrates at $2000 \mathrm{rpm}$ for $30 \mathrm{~s}$ (yielding a PMMA thickness of $\sim 280 \mathrm{~nm}$ ) followed by a soft baking at $170{ }^{\circ} \mathrm{C}$ on a hotplate for $5 \mathrm{~min}$. (iii) Samples were subjected to a $5 \mathrm{~s}$ oxygen plasma $(50 \mathrm{~W}, 250 \mathrm{mTorr}$, Plasma-Therm Batchtop RIE 95m) to enhance the hydrophilicity of the sample surface. (iv) A polyelectrolyte solution (poly diallyldimethylammonium (PDDA) $\mathrm{M}_{\mathrm{W}}=200000 \div 350$ 000, Sigma Aldrich, 0.2 wt. \% in Milli$\mathrm{Q}$ water, Millipore) was pipetted on the surface of the samples and left incubating for $40 \mathrm{~s}$ before rinsing in DI water. This created a positively charged surface layer on the PMMA surface. (v) A suspension of negatively charged polystyrene beads (PS $190 \mathrm{~nm}$ sulfate latex, Interfacial Dynamics Corporation, 0.2 wt. \% in Milli-Q water, Millipore) was added to the surface. The size of the PS beads determines the diameter of the fabricated nanodisks at the end of the processing. After 3 min incubation the suspension was rinsed away using DI water, and the samples were blown dry with nitrogen gas. (vi) A $15 \mathrm{~nm}$ thick Cr film was evaporated using a Lesker PVD 225 
Evaporator at a base pressure of $5 \times 10^{-7}$ Torr, evaporation rate $1 \AA \mathrm{s}^{-1}$. (vii) The PS beads were removed by tape stripping (SWT-10, Nitto Scandinavia AB) for glass, silicon and QCM samples and by a wet tissue for TEM windows. This left a $\mathrm{Cr}$ film with holes at the positions of the stripped PS beads. (viii) The samples were subjected to 5 min oxygen plasma treatment (50 W, 250 mTorr, Plasma-Therm Batchtop RIE 95m) to etch through the PMMA layer exposed beneath the holes in the Cr mask. (ix) Through this mask Pd was deposited at a deposition rate of $1 \AA \mathrm{s}^{-} 1$. To fabricate the PdAu alloys, Au and Pd were deposited in sequence at the same deposition rate through the mask with tailored amount. The thicknesses of each $\mathrm{Au}$ and $\mathrm{Pd}$ layer determined the final composition of the alloy particles. $^{21}$ (x) The remaining PMMA layer was dissolved in acetone in a lift-off step, removing the mask from the sample, only leaving the nanodisk structures on the substrate. (xi) Samples were soaked in IPA and blown dry with nitrogen. (xii) Both $\mathrm{Pd}$ and $\mathrm{Au}-\mathrm{Pd}$ samples were annealed in a homemade flow furnace under $4 \% \mathrm{H}_{2}$ in $\mathrm{Ar}(100$ $\mathrm{ml} \mathrm{min}{ }^{-1}$ ) at $500{ }^{\circ} \mathrm{C}$ for $24 \mathrm{~h}$, except for samples on QCM crystals, which were annealed instead at $400{ }^{\circ} \mathrm{C}$ for $72 \mathrm{~h}$ to avoid $\alpha-\beta$ phase transformation in quartz at $450{ }^{\circ} \mathrm{C}$ that leads to cracking. This way alloying of PdAu samples was achieved.

PTFE deposition. Thin PTFE films were prepared at room temperature in an ultrahigh-vacuum (UHV) DC/RF magnetron sputtering system with a base pressure of $10^{-7}$ mbar and a deposition pressure of $5 \times 10^{-3}$ mbar Ar. ${ }^{42}$ The deposition rate was first determined by sputtering the target independently at a fixed power over a well-defined time interval. The thickness of the reference film was then measured with a DekTak3 profilometer, and the deposition rate $\left(\mathrm{nm} \mathrm{s}^{-1}\right)$ was calculated from the thickness and time.

PMMA deposition. PMMA (MicroChem Corporation, 1 wt. \% diluted in anisole, $\mathrm{M}_{\mathrm{W}}=950$ 000) was spincoated on sensors at $2000 \mathrm{rpm}$ for $30 \mathrm{~s}$ followed by a soft baking at $170{ }^{\circ} \mathrm{C}$ on a hotplate for $5 \mathrm{~min}$ resulted in $35 \mathrm{~nm}$ PMMA film, as measured by ellipsometry (J.A. Woollam M2000). To enable spincoating on an already PTFE-coated sensor to create the tandem structure, a hydrogen plasma treatment was applied for $5 \mathrm{~s}(100 \mathrm{~W}, 250 \mathrm{mTorr}$, Plasma-Therm Batchtop RIE 95m). This step rendered the PTFE surface hydrophilic and thus allowed spincoating of PMMA onto it. The PMMA deposition steps followed the ones described above.

Material characterization. Scanning Electron Microscopy (SEM): All SEM images were obtained in a Zeiss Supra 60 VP with secondary electron detector, working distance $4 \mathrm{~mm}$, and an electron beam acceleration voltage of 10-15 kV. Scanning Transmission Electron Microscopy (STEM) and Energy Dispersive X-ray Spectroscopy (EDS): STEM-EDS measurements were taken using an FEI Titan TEM instrument operated at $300 \mathrm{kV}$ acceleration voltage. The electron probe diameter and convergence semi-angle were approximately $0.15 \mathrm{~nm}$ and $17.5 \mathrm{mrad}$, respectively. HAADF-STEM images were recorded with a collection semi-angle of approximately $47 \mathrm{mrad}$. EDS maps were obtained with a pixel size of $\sim 5 \mathrm{~nm}$ and acquisition time of $1 \mathrm{~s}$ per pixel. The EDS maps were analyzed in Aztec 3.3 (Oxford Instruments). Transmission Kikuchi Diffraction (TKD): The TKD investigation of the samples was performed on an FEI Nova Nano Lab 600 scanning electron microscope (SEM) equipped with Bruker OPTIMUS $^{\text {TM }}$ TKD detector operated at an acceleration voltage of $30 \mathrm{kV}$ and beam current of 1.7 $\mathrm{nA}$ using an aperture of $30 \mu \mathrm{m}$. The electron transparent TEM window containing the nanoparticles was positioned horizontally on the microscope with the particles facing downward, and TKD orientation maps were acquired over a large number of particles with a scan step of 5 $\mathrm{nm}$, and exposure time of approximately $9 \mathrm{~ms}$ per point with a pattern resolution of $320 \times 240$ pixels ( $5 \times 5$ binning). X-ray Photoelectron Spectroscopy (XPS): High-resolution (HR) XPS 
spectra were recorded with a PHI 5000 system (Physical electronics, Chanhassen, Ma, USA) using monochromatized Al Ka radiation $\left(\mathrm{hv}^{1 / 4} 1486.6 \mathrm{eV}\right)$ as the $\mathrm{x}$-ray source. The HR spectra displayed here were obtained at an incident angle of $45^{\circ}$ with a combined energy resolution of $0.01 \mathrm{eV}$. Atomic sensitivity factors for quantitative analysis were taken from a reference. ${ }^{43}$ The binding energies were corrected according to the $\mathrm{Au} 4 \mathrm{f}$ peak $(84 \mathrm{eV})$ and the $\mathrm{C} 1 \mathrm{~s}$ peak $(284.8$ $\mathrm{eV})$. The Au $4 \mathrm{f}$ peak was also employed to correct for any additional charge build during HR XPS measurements. For all measurements, the samples were electrically grounded to prevent accumulation of electrostatic charge. Atomic Force Microscopy (AFM): AFM measurements were performed in tapping mode using an SPM Bruker Dimension 3100 system in air. In situ $X$ ray Diffraction $(X R D)$ : The in situ XRD measurements were performed with a Bruker D8 Advance $(\mathrm{Co}-\mathrm{K} \alpha \lambda=0.1789 \mathrm{~nm})$ that is equipped with an LYNXEYE 1D detector. During the XRD measurements, the sample was hydrogenated at a constant temperature of $25{ }^{\circ} \mathrm{C}$ inside an Anton Paar XRK 900 reactor chamber. As the loading gas, a mixture of $96.0 \%$ helium and $4.0 \%$ $\mathrm{H}_{2}$ was used and a constant flow of at least $20 \mathrm{sccm}$ was maintained at all times. After setting and reaching a new pressure set point, we waited 10 min before commencing the XRD measurements to make sure that the sample fully responded to the new experimental conditions. The XRD diffractograms are background corrected by subtracting the diffractogram of an empty substrate. The values for the d111-spacing (the out-of-plane direction of the film) and the FWHM were obtained from the best fit of a pseudo-Voigt function to the background-corrected experimental data. The rocking curves were collected around the $<111>$ diffraction peak with a Bruker D8 Discover diffractometer $(\mathrm{Cu}-\mathrm{K} \alpha, \lambda=0.1541 \mathrm{~nm})$. This diffractometer was also used for the $e x$ situ XRD measurements. Fourier Transform Infrared Spectroscopy (FTIR): Bruker IFS66 spectrometer with $\mathrm{KBr}$ windows was used. The samples were mounted in a cell which can be pumped and filled with hydrogen at room temperature. A $200 \mathrm{~nm}$ PTFE layer was sputtered on a $10 \mathrm{~nm}$ Pd film on a Kbr substrate. The films was measured in the as-deposited state and after exposure to hydrogen for $10 \mathrm{~min}$.

Hydrogen sensing measurements. Hydrogenation Isotherm, Kinetics and LoD Measurements: All isotherm and kinetics measurements were performed in an in-house-made vacuum chamber setup with optical windows reported earlier. ${ }^{4,21}$ The absolute hydrogen pressure in the chamber was monitored using two capacitive pressure gauges with different range (MKS Baratron). Optical transmittance measurements through the sample were enabled by UHV-compatible sapphire windows mounted on the vacuum chamber, and by using a fiber-coupled, unpolarized white light source (AvaLight-Hal, Avantes) and a fixed grating fiber coupled spectrophotometer (SensLine AvaSpec-2048XL, Avantes). The pressure inside the chamber was controlled using a microbar-precision leak valve. The temperature was maintained with a heating coil wrapped around the chamber and a temperature controller (Eurotherm $3216 \mathrm{~N}$ ) in a feedback loop manner, where the sample surface temperature inside the vacuum chamber was continuously used as input. All experiments were performed at constant $30{ }^{\circ} \mathrm{C}$. The $\mathrm{LoD}$ measurements were performed at $1 \mathrm{~Hz}$ sampling frequency $(0.165 \mathrm{~ms}$ integration time with 1000 averages $)$. The kinetics measurements were performed at $6 \mathrm{~Hz}$ frequency $(0.165 \mathrm{~ms}$ integration time with 10 averages). The observed higher noise in the kinetics data is thus a consequence of the lower number of averaged spectra per data point (Fig. S22). It is also important to note that the apparent sampling frequency obtained by the setup is slower than the theoretical one (i.e. integration time $\times$ averaging number) due to the delay in the computing system when acquiring, processing, analyzing and plotting the data in real time. The LSPR peak descriptors were obtained by fitting a Lorentzian-function to the wavelength range at $\pm 60 \mathrm{~nm}$ around the LSPR 
peak in the measured optical extinction spectra, within which a good fit $\left(\mathrm{R}^{2}>0.95\right)$ is obtained, despite the asymmetry of the global LSPR peak (Fig. S19). In the experiments to determine the apparent activation energies, absorption and desorption kinetics measurements to and from 900 mbar were performed at four different temperatures, i.e. $30-60{ }^{\circ} \mathrm{C}$, in $10{ }^{\circ} \mathrm{C}$ steps. For the Arrhenius analysis, $t_{50}$ was employed as a measure of the process rate. For the long-term stability test, we stored the $\mathrm{Pd}$ sensors at controlled ambient conditions (average temperature $21.2{ }^{\circ} \mathrm{C}$, humidity $23 \% \mathrm{RH}, \mathrm{CO}_{2} 323 \mathrm{ppm}$ ). Furthermore, we intentionally performed the test on pure $\mathrm{Pd}$ sensors (and not on the $\mathrm{Pd}_{70} \mathrm{Au}_{30}$ alloy) for two reasons: (i) the neat $\mathrm{Pd}$ sensor exhibits much slower response than the analogue $\mathrm{Pd}_{70} \mathrm{Au}_{30}$ and thus its kinetics can be resolved in more detail so that any variation due to measurement artifacts can be eliminated. (ii) Neat Pd is significantly more prone to poisoning than $\mathrm{PdAu}$ (see Fig. S50 and also e.g. ref. 44), which makes it a more effective system to investigate the long-term stability of a polymer coating with respect to preventing sensor deactivation. Deactivation and Poisoning Tests and LoD Measurements in Ar and Air: The deactivation and LoD measurements were carried out in a quartz tube flow reactor at atmospheric pressure with optical access for transmittance measurements (X1, Insplorion AB), using synthetic air as the carrier gas, as well as Ar for the LoD experiment. ${ }^{44}$ The gas flow rate (kept constant at 100 and 500 or $325 \mathrm{~mL} \mathrm{~min}^{-1}$, for deactivation and LoD measurements in Ar and air, respectively) and gas composition were regulated by mass flow controllers (Bronkhorst $\Delta \mathrm{P})$. The sample inside the flow reactor was illuminated by white light (AvaLight-Hal, Avantes) through an optical fiber equipped with a collimating lens. The transmitted light was then analyzed using a fiber-coupled fixed-grating spectrometer (AvaSpec-1024, Avantes, or SensLine AvaSpec-2048XL, Avantes, for LoD measurements). The measurement temperature was maintained at $30{ }^{\circ} \mathrm{C}$. Quartz Crystal Microbalance Measurements: The QCM window-module (QSense Explorer Microscopy, Biolin Scientific Corporation) was connected to a series of mass flow controllers (Bronkhorst $\Delta \mathrm{P}$ ) to regulate the $\mathrm{H}_{2}$ partial pressure in Ar carrier gas at a constant total flow rate of $30 \mathrm{~mL} \mathrm{~min}{ }^{-1}$. Data collection was executed by QSoft ${ }^{\circledR}$ (Biolin Scientific AB). The absorbed hydrogen content in the nanoparticles was calculated from the QCM frequency shift via the Sauerbrey equation ${ }^{45}$ and analysis of the nanoparticle surface coverage based on SEM images of the crystal, as described in detail elsewhere. ${ }^{22}$ All of the measurements were carried out at $30{ }^{\circ} \mathrm{C}$.

Density Functional Theory calculations. All the modeled structures were optimized within the DFT framework. The cluster models were optimized using the PBE functional ${ }^{46}$ while van der Waals contributions were included for the polymer and polymer- $\mathrm{H}_{2}$ systems by using the vdWCX functional. ${ }^{47}$ Geometry optimizations were performed using the Vienna ab initio simulation package, VASP. ${ }^{48,49}$ Projector augmented waves $(\mathrm{PAW})^{50,51}$ were used, with a plane-wave kinetic energy cutoff of $500 \mathrm{eV}$. To avoid artificial interaction due to the periodic boundaries conditions, a $30 \times 30 \times 31 \AA$ supercell was used, ensuring at least $16 \AA$ of vacuum between two successive images. $\Gamma$-centered ${ }^{52}$ calculations were performed with a Gaussian smearing, $\sigma$, of $0.02 \mathrm{eV}$, with the energies being extrapolated to $\sigma=0.00 \mathrm{eV}$. The atoms positions were optimized until the criterion of the residual forces on any direction being less than $0.02 \mathrm{eV} \AA^{-1}$ was met.

Finite-Difference Time-Domain simulations. We used FDTD Solutions to calculate the optical spectra of a Pd nanodisk as a function of the refractive index of the coating, as well as coating thickness. Due to the fact that the fabricated arrays are amorphous in terms of the nanoparticle arrangement on the surface, we cannot model exactly the whole sensor and accordingly are limited to either a single disk or, to account for inter-particle coupling, a periodic lattice. In the 
present case we first chose the former solution, although in one case we also consider a hexagonal lattice. The Pd nanodisk is modeled with a diameter of $180 \mathrm{~nm}, 30 \mathrm{~nm}$ thickness and rounded edges. The permittivity of $\mathrm{Pd}$ and $\mathrm{Pd}$ hydride $\left(\mathrm{PdH}_{0.67}\right)$ are taken from the literature. ${ }^{53}$ The structures are placed directly on top of a glass substrate $(\mathrm{n}=1.45)$ and are conformally coated by an isotropic dielectric layer with a variable thickness and refractive index (RI). In the simulations, we span the thickness in the range from 5 to $75 \mathrm{~nm}$ and the RI from 1 to 1.6. This encompasses the range of the parameters considered in the experiment. The structures are illuminated by a plane wave and extinction is collected and analyzed in terms of resonance position.

\section{References}

1. Hydrogen to the rescue. Nature Materials 17, 565 (2018).

2. U.S. Department of Energy, Energy Efficiency and Renewable Energy (EERE), Fuel Cell Technologies Office. Multi-Year Research, Development, and Demonstration Plan, 2011 2020. Section 3.7 Hydrogen Safety, Codes and Standards. (2015).

3. Wadell, C., Syrenova, S. \& Langhammer, C. Plasmonic hydrogen sensing with nanostructured metal hydrides. ACS Nano 8, 11925-11940 (2014).

4. Wadell, C. et al. Hysteresis-Free Nanoplasmonic Pd-Au Alloy Hydrogen Sensors. Nano Lett. 15, 3563-3570 (2015).

5. Yip, H. K. et al. Gold Nanobipyramid-Enhanced Hydrogen Sensing with Plasmon Red Shifts Reaching $\approx 140 \mathrm{~nm}$ at 2 vol\% Hydrogen Concentration. Adv. Opt. Mater. 5, 1700740 (2017).

6. Liu, N., Tang, M. L., Hentschel, M., Giessen, H. \& Alivisatos, A. P. Nanoantennaenhanced gas sensing in a single tailored nanofocus. Nat. Mater. 10, 631-636 (2011).

7. Tittl, A. et al. Palladium-Based Plasmonic Perfect Absorber in the Visible Wavelength Range and Its Application to Hydrogen Sensing. Nano Lett. 11, 4366-4369 (2011).

8. Langhammer, C., Zorić, I., Kasemo, B. \& Clemens, B. M. Hydrogen storage in Pd nanodisks characterized with a novel nanoplasmonic sensing scheme. Nano Lett. 7, 31223127 (2007).

9. Sterl, F. et al. Magnesium as Novel Material for Active Plasmonics in the Visible Wavelength Range. Nano Lett. 15, 7949-7955 (2015).

10. Baldi, A., Narayan, T. C., Koh, A. L. \& Dionne, J. A. In situ detection of hydrogeninduced phase transitions in individual palladium nanocrystals. Nat. Mater. 13, 1143-1148 (2014).

11. Syrenova, S. et al. Hydride formation thermodynamics and hysteresis in individual Pd nanocrystals with different size and shape. Nat. Mater. 14, 1236-1244 (2015).

12. Favier, F., Walter, E. C., Zach, M. P., Benter, T. \& Penner, R. M. Hydrogen sensors and switches from electrodeposited palladium mesowire arrays. Science (80-. ). 293, 22272231 (2001).

13. Penner, R. M. A Nose for Hydrogen Gas: Fast, Sensitive H2 Sensors Using 
Electrodeposited Nanomaterials. Acc. Chem. Res. 50, 1902-1910 (2017).

14. Adams, B. D. \& Chen, A. The role of palladium in a hydrogen economy. Mater. Today 14, 282-289 (2011).

15. Poyli, M. A. et al. Multiscale Theoretical Modeling of Plasmonic Sensing of Hydrogen Uptake in Palladium Nanodisks. J. Phys. Chem. Lett. 3, 2556-2561 (2012).

16. Schwarz, R. B. \& Khachaturyan, A. G. Thermodynamics of open two-phase systems with coherent interfaces: Application to metal-hydrogen systems. Acta Mater. 54, 313-323 (2006).

17. Hübert, T., Boon-Brett, L., Black, G. \& Banach, U. Hydrogen sensors - A review. Sensors Actuators, B Chem. 157, 329-352 (2011).

18. Palmisano, V. et al. Selectivity and resistance to poisons of commercial hydrogen sensors. Int. J. Hydrogen Energy 40, 11740-11747 (2015).

19. Clerbaux, C. et al. Carbon monoxide pollution from cities and urban areas observed by the Terra/MOPITT mission. Geophys. Res. Lett. 35, L03817 (2008).

20. Luo, S., Wang, D. \& Flanagan, T. B. Thermodynamics of Hydrogen in fcc Pd-Au Alloys. J. Phys. Chem. B 114, 6117-6125 (2010).

21. Nugroho, F. A. A., Iandolo, B., Wagner, J. B. \& Langhammer, C. Bottom-Up Nanofabrication of Supported Noble Metal Alloy Nanoparticle Arrays for Plasmonics. ACS Nano 10, 2871-2879 (2016).

22. Nugroho, F. A. A., Darmadi, I., Zhdanov, V. P. \& Langhammer, C. Universal Scaling and Design Rules of Hydrogen-Induced Optical Properties in Pd and Pd-Alloy Nanoparticles. ACS Nano 12, 9903-9912 (2018).

23. Mayer, K. M. \& Hafner, J. H. Localized surface plasmon resonance sensors. Chem. Rev. 111, 3828-3857 (2011).

24. Fukai, Y. The Metal-Hydrogen System. (Springer-Verlag, 1993).

25. Zoric', I., Larsson, E. M., Kasemo, B. \& Langhammer, C. Localized Surface Plasmons Shed Light on Nanoscale Metal Hydrides. Adv. Mater. 22, 4628-4633 (2010).

26. Li, G. et al. Hydrogen storage in Pd nanocrystals covered with a metal-organic framework. Nat. Mater. 13, 802-806 (2014).

27. Ngene, P. et al. Polymer-Induced Surface Modifications of Pd-based Thin Films Leading to Improved Kinetics in Hydrogen Sensing and Energy Storage Applications. Angew. Chemie Int. Ed. 53, 12081-12085 (2014).

28. Pivak, Y., Schreuders, H., Slaman, M., Griessen, R. \& Dam, B. Thermodynamics, stress release and hysteresis behavior in highly adhesive $\mathrm{Pd}-\mathrm{H}$ films. Int. J. Hydrogen Energy 36, 4056-4067 (2011).

29. Yoo, H.-W., Cho, S.-Y., Jeon, H.-J. \& Jung, H.-T. Well-Defined and High Resolution Pt Nanowire Arrays for a High Performance Hydrogen Sensor by a Surface Scattering Phenomenon. Anal. Chem. 87, 1480-1484 (2015).

30. Yang, F., Kung, S.-C., Cheng, M., Hemminger, J. C. \& Penner, R. M. Smaller is Faster 
and More Sensitive: The Effect of Wire Size on the Detection of Hydrogen by Single Palladium Nanowires. ACS Nano 4, 5233-5244 (2010).

31. Koo, W.-T. et al. Accelerating Palladium Nanowire H2 Sensors Using Engineered Nanofiltration. ACS Nano 11, 9276-9285 (2017).

32. Delmelle, R., Ngene, P., Dam, B., Bleiner, D. \& Borgschulte, A. Promotion of Hydrogen Desorption from Palladium Surfaces by Fluoropolymer Coating. ChemCatChem 8, 16461650 (2016).

33. Nanba, Y., Tsutsumi, T., Ishimoto, T. \& Koyama, M. Theoretical Study of the Hydrogen Absorption Mechanism into a Palladium Nanocube Coated with a Metal-Organic Framework. J. Phys. Chem. C 121, 14611-14617 (2017).

34. Langhammer, C., Zhdanov, V. P., Zorić, I. \& Kasemo, B. Size-Dependent Kinetics of Hydriding and Dehydriding of Pd Nanoparticles. Phys. Rev. Lett. 104, 135502 (2010).

35. Jeon, K.-J. et al. Air-stable magnesium nanocomposites provide rapid and high-capacity hydrogen storage without using heavy-metal catalysts. Nat. Mater. 10, 286-290 (2011).

36. Hong, J. et al. A highly sensitive hydrogen sensor with gas selectivity using a PMMA membrane-coated Pd nanoparticle/single-layer graphene hybrid. ACS Appl. Mater. Interfaces 7, 3554-3561 (2015).

37. Min, K. E. \& Paul, D. R. Effect of tacticity on permeation properties of poly(methyl methacrylate). J. Polym. Sci. Part B Polym. Phys. 26, 1021-1033 (1988).

38. ISO 26142:2010 Hydrogen detection apparatus - Stationary applications. (2010).

39. Yamada, Y., Yamada, T., Tasaka, S. \& Inagaki, N. Surface Modification of Poly(tetrafluoroethylene) by Remote Hydrogen Plasma. Macromolecules 29, 4331-4339 (1996).

40. Fredriksson, H. et al. Hole-Mask Colloidal Lithography. Adv. Mater. 19, 4297-4302 (2007).

41. Grant, A. W., Hu, Q.-H. \& Kasemo, B. Transmission electron microscopy windows for nanofabricated structures. Nanotechnology 15, 1175-1181 (2004).

42. Slaman, M., Westerwaal, R., Schreuders, H. \& Dam, B. Optical hydrogen sensors based on metal-hydrides. in Proceedings SPIE 8368, 836805 (International Society for Optics and Photonics, 2012).

43. Briggs, D. \& Seah, M. P. Practical Surface Analysis, Auger and X-ray Photoelectron Spectroscopy. (Wiley, 1990).

44. Nugroho, F. A. A. et al. Plasmonic Nanospectroscopy for Thermal Analysis of Organic Semiconductor Thin Films. Anal. Chem. 89, 2575-2582 (2017).

45. Sauerbrey, G. Verwendung von Schwingquarzen zur Wägung dünner Schichten und zur Mikrowägung. Zeitschrift für Phys. 155, 206-222 (1959).

46. Perdew, J. P., Burke, K. \& Ernzerhof, M. Generalized Gradient Approximation Made Simple. Phys. Rev. Lett. 77, 3865-3868 (1996).

47. Berland, K. \& Hyldgaard, P. Exchange functional that tests the robustness of the plasmon 
description of the van der Waals density functional. Phys. Rev. B 89, 035412 (2014).

48. Kresse, G. \& Furthmüller, J. Efficiency of ab-initio total energy calculations for metals and semiconductors using a plane-wave basis set. Comput. Mater. Sci. 6, 15-50 (1996).

49. Kresse, G. \& Furthmüller, J. Efficient iterative schemes for ab initio total-energy calculations using a plane-wave basis set. Phys. Rev. B 54, 11169-11186 (1996).

50. Blöchl, P. E. Projector augmented-wave method. Phys. Rev. B 50, 17953-17979 (1994).

51. Kresse, G. \& Joubert, D. From ultrasoft pseudopotentials to the projector augmented-wave method. Phys. Rev. B 59, 1758-1775 (1999).

52. Monkhorst, H. J. \& Pack, J. D. Special points for Brillouin-zone integrations. Phys. Rev. B 13, 5188-5192 (1976).

53. Silkin, V. M., Díez Muĩo, R., Chernov, I. P., Chulkov, E. V \& Echenique, P. M. Tuning the plasmon energy of palladium-hydrogen systems by varying the hydrogen

concentration. J. Phys. Condens. Matter 24, 104021 (2012).

\section{Acknowledgments}

We acknowledge financial support from the Swedish Foundation for Strategic Research Framework project RMA15-0052, the Knut and Alice Wallenberg Foundation project 2016.0210, and the Polish National Science Center project 2017/25/B/ST3/00744. We also thank the Knut and Alice Wallenberg Foundation for their support of the infrastructure in the MC2 nanofabrication laboratory at Chalmers. The electronic structure calculations were performed on resources provided by the Swedish National Infrastructure for Computing at NSC and C3SE (projects SNIC2017-1-632, SNIC2017-12-18 and C3SE2018-1-6). We thank Joachim Fritzsche for the help with the SEM figure and Martin Slaman (VU Amsterdam) for FTIR measurements.

\section{Author contributions}

F.A.A.N. and C.L. designed the experiments, analyzed the data and wrote the manuscript. F.A.A.N. and I.D. fabricated the sensors. F.A.A.N. performed sensing measurements on PTFE sensors. F.A.A.N. and I.D. performed sensing measurements on PMMA and tandem sensors. L.C. and A.H. executed the DTF calculations. A.S.-A. performed the XPS analysis. H.S. deposited the PTFE thin films. L.J.B. and B.D. performed the XRD analysis. A.B.d.S.F. and J.B.W. performed the TKD analysis. S.K. performed the STEM-EDS analysis. T.J.A. performed the FDTD simulations. V.P.Z. contributed the theoretical analysis on the sensor kinetics and PTFE strain. B.D. and C.L. coined the initial idea. C.L. coordinated the project.

\section{Additional information}

Supplementary Information is available in the online version of the paper. Reprints and permissions information is available online at www.nature.com/reprints.

Correspondence and requests for materials should be addressed to F.A.A.N. or C.L. 


\section{Competing financial interests}

The authors declare no competing financial interests. 